\title{
Lumbar Puncture: Indications, Challenges and Recent Advances
}

\author{
Biswamohan Mishra and Venugopalan Y Vishnu \\ Department of Neurology, All India Institute of Medical Sciences, New Delhi, India.
}

DOI: https://doi.org/10.17925/USN.2021.17.1.23

$\mathrm{T}$ he methodological, diagnostic and therapeutic aspects of lumbar puncture and cerebrospinal fluid (CSF) study have advanced leaps and bounds since its conception in 1890 by Quincke. CSF study is commonly used to diagnose CSF infections, but technological advances have enabled it to be used to diagnose many autoimmune, neuroinflammatory diseases of the central nervous system. CSF biomarkers are an important tool in diagnosing some neurodegenerative diseases; recent improvements in detecting these biomarkers may aid in developing drugs for these chronic, progressive, disabling diseases. Patients with diseases like spinal muscular atrophy, who were only treated supportively for decades, can now benefit from intrathecal nusinersen. Lumbar puncture is generally a safe intervention, yet complications can occur, ranging from mild and self-limiting, e.g. low backache, to severe, e.g. cerebral and spinal coning. Meta-analyses, consensus guidelines and systematic reviews have shown that using an atraumatic needle tip results in fewer complications, and guidelines emphasize the need to increase awareness among physicians and change current practice. Demand for image-guided lumbar puncture has increased significantly since 2000. Deferring lumbar puncture until after brain magnetic resonance imaging may be detrimental in situations where performing lumbar puncture is vital. Certain clinical features should warrant advanced imaging before lumbar puncture in suspected meningitis, instead of routinely performing imaging in every patient before lumbar puncture.

\section{Keywords}

Lumbar puncture, spinal puncture, cerebrospinal fluid, post-dural puncture headache, post-lumbar puncture headache, checklist Lumbar puncture

Disclosures: Biswamohan Mishra and Venugopalan Y Vishnu have no financial or non-financial relationships or activities to declare in relation to this article.

Acknowledgements: The authors acknowledge Ms Abhilasha Krishnamurthy (December Design Studio) for designing the figures.

Review process: Double-blind peer review.

Compliance with ethics: This article involves a review of literature and does not report on new clinical data, or any studies with human or animal subjects performed by any of the authors.

Authorship: All named authors meet the criteria of the International Committee of Medical Journal Editors for authorship for this manuscript, take responsibility for the integrity of the work as a whole and have given final approval for the version to be published.

Access: This article is freely accessible at

touchNEUROLOGY.com @Touch Medical Media 2021

Received: 25 October 2020

Accepted: 21 April 2021

Published online: 2 August 2021

Citation: touchREVIEWS in Neurology. 2021;17(1):23-31

Corresponding author: Venugopalan Y Vishnu,

Room No. 704, Cardiothoracic and Neurosciences

Centre, All India Institute of Medical Sciences, New

Delhi, India. PIN - 110029. E: vishnuvy16@yahoo.com

Support: No funding was received for

the publication of this article.
Studying cerebrospinal fluid (CSF) is essential for diagnosing many central nervous system (CNS) diseases, including infection, inflammation and malignancy. Lumbar puncture is a relatively safe and routinely performed procedure for extracting CSF.' In this review, we summarize the essential CSF flow dynamics, common indications and contraindications for lumbar puncture, and describe the procedures to rule out these contraindications. We propose a checklist before undertaking lumbar puncture, describe trends regarding the primary providers of lumbar puncture procedure since the 2000s, discuss the feasibility of outpatient lumbar puncture and outline the complications of lumbar puncture. We also address some long-standing myths about lumbar puncture, and propose a flow chart to approach difficult and failed lumbar punctures. The objectives of this review article are:

- to describe the common indications, contraindications and complications of lumbar puncture

- to describe the procedures that can be used to avoid these complications, and propose a safety checklist based on available evidence

- to study the recent trends regarding the primary providers of lumbar puncture

- to describe the diagnostic and therapeutic advancements of lumbar puncture

- to assess the feasibility and safety of outpatient lumbar puncture

- to review the evidence for some commonly performed clinical practices relating to lumbar puncture.

With the keywords "lumbar puncture", "spinal tap" and "CSF examination", we searched in Google Scholar, Embase, PubMed, Medline and the Cochrane Library for relevant articles, case reports, case series, meta-analyses and systematic reviews. We also reviewed published consensus guidelines and expert opinions. The references cited in the reviews were also analysed. Search terms included combinations of the Medical Subject Headings "spinal puncture", "meningitis", "cerebrospinal fluid", "injections, spinal", "central nervous system", "practice guideline", "prevention and control", "post-dural post-lumbar puncture headache", "trends", "check list", "outpatient", "complication", "meningeal enhancement post-LP", "CT prior to LP", "difficult LP" and "biomarkers/cerebrospinal fluid".

\section{Cerebrospinal fluid flow dynamics}

Most CSF is formed by the choroid plexus of the ventricles at a rate of approximately $0.35 \mathrm{~cm}^{3} / \mathrm{min}$, or $500 \mathrm{~cm}^{3} /$ day, which is three to four times the total volume of CSF. ${ }^{2}$ The ependymal surfaces of the ventricles and arachnoid membranes also produce small amounts. The red arrows in Figure 1 show the main channels of CSF flow. The CSF secreted in the lateral ventricles passes first into the third ventricle, then downwards through the aqueduct of Sylvius into the fourth ventricle. CSF from the fourth ventricle flows laterally through the foramen of Luschka and the foramen of Magendie, medially to the subarachnoid space. Egress of CSF through the foramen of Magendie results in the filling of the 
Figure 1: Schematic of cerebrospinal fluid flow dynamics

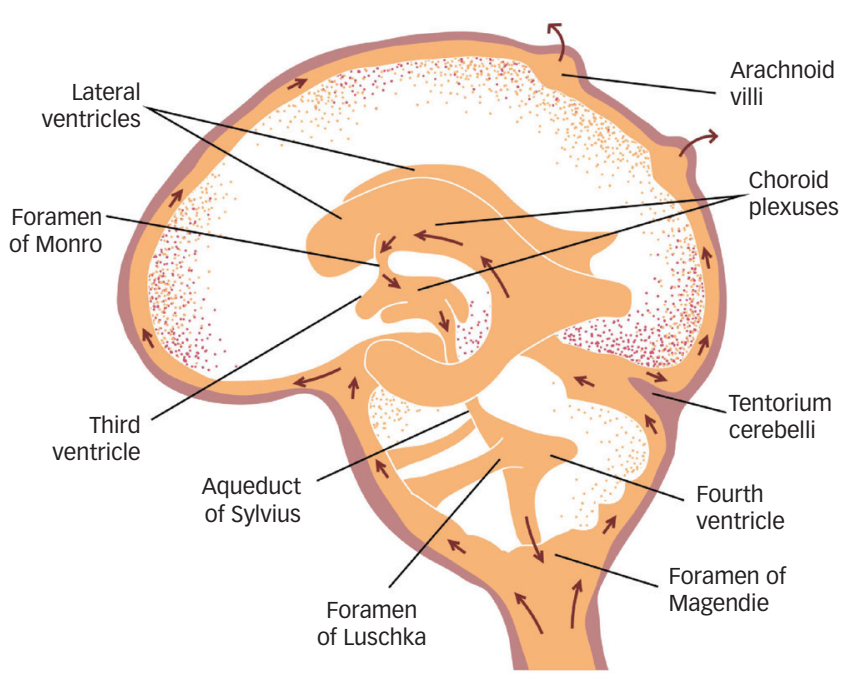

The red arrows show the pathway of CSF flow from the choroid plexus in the lateral ventricles to the arachnoid villi protruding into the dural sinuses. The light orange colour depicts the cerebrospinal fluid surrounding the brain structures.

spinal subarachnoid space, from where CSF is drained by lumbar puncture. CSF draining through the foramen of Luschka flows into the cisterna magna, then upwards into the subarachnoid space overlying the cerebral cortex. From there it is absorbed into the venous sinuses through the arachnoid villi and finally returned to systemic circulation (Figure 1). ${ }^{3}$ The $10-15 \mathrm{~cm}^{3}$ of CSF collected during lumbar puncture is replenished in approximately half an hour in a healthy, normally hydrated patient. ${ }^{4}$

\section{Common indications for lumbar puncture}

CNS infections continue to be the major indication for diagnostic lumbar puncture. ${ }^{5}$ Normal CSF is clear, with: opening pressure (OP) 10-20 $\mathrm{cm} \mathrm{H} \mathrm{H}_{2} \mathrm{O}$, white blood cell concentration $<5$ cells $/ \mu \mathrm{L}$, proteins $<40 \mathrm{mg} / \mathrm{dL}$, glucose $26-45 \mathrm{mg} / \mathrm{dL}$ and CSF:serum glucose ratio $>0.66$. In bacterial meningitis, CSF is turbid, cloudy or purulent. In addition, OP is raised, alongside raised white blood cell count (usually $>100$ cells $/ \mu \mathrm{L}$, predominantly neutrophils), raised protein (>100 mg/dL), low glucose, and very low CSF:serum glucose ratio. CSF in viral meningitis is clear, with normal or mildly raised OP, raised white blood cell count (normal range $<1,000$ cells/ $\mu \mathrm{L}$, predominantly lymphocytes), mildly raised protein (50-100 mg/dL), normal or slightly low glucose, and normal or slightly low CSF:serum glucose ratio. ${ }^{6}$ In tuberculous meningitis, CSF is clear or cloudy, with raised OP, raised white blood cell count (normal range $<500$ cells/ML, predominantly lymphocytes), highly raised proteins, low glucose and very low CSF:serum glucose ratio. ${ }^{6}$ CSF lactate and procalcitonin tests may indicate a bacterial cause, but further accuracy tests are required before these can be recommended for routine use. Gram stain and culture help to identify the organism and assess antimicrobial susceptibilities. Multiplex polymerase chain reaction $(\mathrm{PCR})^{7}$ and matrix-assisted laser desorption/ionization-time of flight $(\mathrm{MALDI}-\mathrm{TOF})^{8}$ are newer methods that can identify organisms from CSF more quickly than existing methods.

Other than meningitis, CSF is essential in diagnosing some neuroinflammatory conditions, such as primary CNS vasculitis, ${ }^{9}$ autoimmune encephalitis, ${ }^{10}$ acute transverse myelitis ${ }^{11}$ and GuillainBarré syndrome. ${ }^{12}$ For N-methyl-D-aspartate receptor (anti-NMDAR) encephalitis, CSF is more sensitive than serum for detecting NMDAR antibodies. ${ }^{10} \mathrm{CSF}$ is abnormal in $80-90 \%$ of patients with primary CNS vasculitis, namely a mildly increased leukocyte count and total protein concentration. ${ }^{9}$ In inflammatory causes of transverse myelitis, CSF is abnormal in approximately one-half of patients, with a moderate lymphocytosis (typically $<100$ cells/ $\mathrm{LL}$ ) and an elevated protein level (usually $100-120 \mathrm{mg} / \mathrm{dL}$ ). Glucose levels are usually normal. ${ }^{13}$ Additional CSF studies in patients with transverse myelitis include venereal disease research laboratory tests, oligoclonal bands (OCBS), IgG index, cytology and further studies targeting a particular organism (if an infectious aetiology is suspected). ${ }^{13}$ CSF OCBS are positive in $85-95 \%$ of patients with multiple sclerosis, and their absence in CSF has a strongly negative predictive value of $90 \%$, helping to distinguish multiple sclerosis from other inflammatory demyelinating diseases, such as neuromyelitis optica spectrum disorders and myelin oligodendrocyte glycoprotein associated disease. ${ }^{14}$ CSF can be used to confirm the diagnosis of subarachnoid haemorrhage when the results of brain imaging are inconclusive, by analysing degraded haemoglobin particles in CSF. ${ }^{15}$ CSF cytology can identify suspected leptomeningeal metastases, and CSF flowcytometry can corroborate the diagnosis of CNS lymphoma. ${ }^{16}$

An OP $>25 \mathrm{~cm} \mathrm{H}_{2} \mathrm{O}$ is essential for diagnosing idiopathic intracranial hypertension. ${ }^{17} \mathrm{~A}$ normal $\mathrm{OP}$ and improvement in gait after removal of 30-50 $\mathrm{mL}$ of CSF confirms the diagnosis of normal pressure hydrocephalus and predicts the efficacy of CSF catheter placement. ${ }^{18}$

Lumbar puncture can also be used therapeutically to administer drugs intrathecally, e.g. colistin and vancomycin in ventriculitis, ${ }^{19}$ interferon in subacute sclerosing panencephalitis, ${ }^{20}$ nusinersen (Spinraza ${ }^{\oplus}$, Biogen, Cambridge, MA, USA) in spinal muscular atrophy, ${ }^{21}$ and chemotherapy ${ }^{22}$ and baclofen for spasticity. ${ }^{23}$ Perioperative intrathecal fluorescein helps visualization of CSF leaks at the skull base. ${ }^{24}$ In other disorders, lumbar puncture is itself a treatment. In acute communicating hydrocephalus and cryptococcal meningitis, headache caused by raised intracranial pressure can be relieved by draining CSF through lumbar puncture, ${ }^{25}$ which is associated with a $69 \%$ relative improvement in survival. ${ }^{26}$ In patients with idiopathic intracranial hypertension and imminent visual loss, lumbar puncture can be used as a rescue measure to save vision before other definitive CSF diversion procedures can be done. ${ }^{27}$

\section{Contraindications for lumbar puncture}

Lumbar puncture has several contraindications, which can be categorized as absolute and relative contraindications. The following are the absolute contraindications:

- non-communicating obstructive hydrocephalus ${ }^{28}$

- cerebral mass lesion causing brain shift ${ }^{29}$

- spinal cord compression ${ }^{30}$

- skin infection near the lumbar puncture site (e.g. suspected spinal epidural abscess) ${ }^{31}$

- congenital anomalies, such as Chiari malformations, tethered spinal cord and myelomeningocele. ${ }^{32}$

The major relative contraindications include low platelet count and the use of antiplatelets and anticoagulants. ${ }^{32}$ There is still controversy as to the lowest platelet count at which lumbar puncture can be done safely to avoid causing spinal or epidural haematoma. Consensus guidelines propose that a platelet count of higher than $40 \times 10^{\%} / \mathrm{L}$ in adults before an elective spinal tap is safe (based on level 3 evidence). ${ }^{32}$

Use of antiplatelets and anticoagulants also constitutes a relative contraindication. These antithrombotic drugs, except argatroban, are 
Figure 2: Recommended time interval for discontinuing commonly used antiplatelet and anticoagulant medications before lumbar puncture

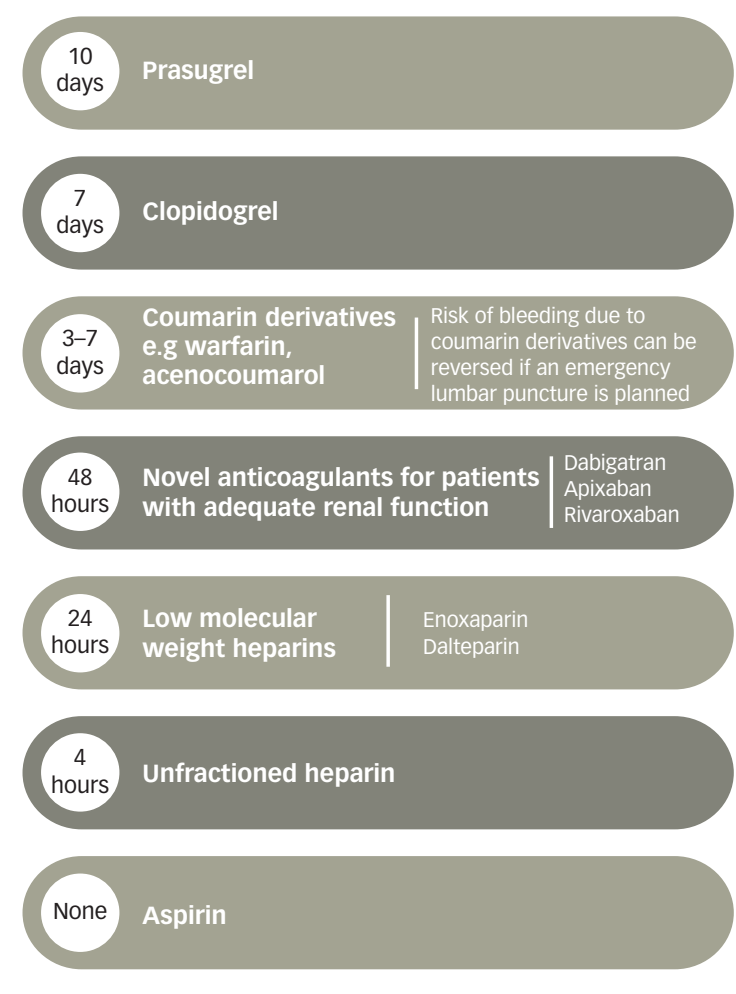

cleared through the kidneys, and therefore their serum concentrations may be higher in patients with impaired renal function. ${ }^{33}$ Figure 2 shows the discontinuation schedule proposed by The European Society of Anaesthesiology and Intensive Care for antiplatelet and anticoagulant medications prior to lumbar puncture, to mitigate the risk of haemorrhagic complications (e.g. epidural haematoma). ${ }^{33}$ To address this issue further, Lee et al. studied 665 patients receiving single or dual antiplatelet therapy who underwent lumbar puncture. Patients were stratified by time of antiplatelet drug discontinuation before lumbar puncture: $<1$ week, 1-4 weeks, and $>4$ weeks. There was no meaningful increase in haemorrhagic complications in patients receiving aspirin, clopidogrel or both, regardless of how early the antiplatelet drug was discontinued. Antiplatelet use within 1 week was associated with an incidence of $3 \%$ and $4 \%$ risk for bloody and traumatic lumbar puncture, respectively. The incidence of bloody and traumatic lumbar puncture for the 1-4 weeks group was $2.2 \%$ and $4.5 \%$, respectively. For the $>4$ weeks group, the incidence was $5 \%$ for both bloody and traumatic lumbar puncture. The rate of haematoma complications was highest $(0.7 \%)$ in patients receiving aspirin at the time of the procedure..$^{34}$

In a recent study by Bodilsen et al., a population-based cohort was assessed using medical registries to determine the risk of spinal haematoma after lumbar puncture in patients with and without coagulopathy. Coagulopathy was defined as platelet count lower than $150 \times 10^{\%} / \mathrm{L}$, international normalized ratio $>1.4$, or activated partial thromboplastin time $>39 \mathrm{~s}$. In total, 83,711 lumbar puncture procedures in 64,730 patients were analysed. The risk of spinal haematoma following lumbar puncture was $0.20 \%(99 / 49,526)$ among patients without coagulopathy and $0.23 \%(24 / 10,371)$ among those with coagulopathy, suggesting no meaningful difference. However, selection bias by physicians in selecting relatively low-risk patients for lumbar puncture could not be ruled out. ${ }^{35}$

\section{Procedures to rule out contraindications for lumbar puncture}

Lumbar puncture has several contraindications, most of which can be ruled out by other procedures. We propose performing the procedures outlined in Figure 3 , in order to rule out the conditions that contraindicate.

\section{Trends in lumbar puncture over primary lumbar puncture procedure providers since the 1990s}

With the advent of more complex surgeries and costlier medications, the duty of performing the lumbar puncture has shifted from general practitioner to radiologist since the 1990s. Fluoroscopy-guided and ultrasound-guided lumbar puncture are becoming more sought after..$^{36}$ Kroll et al. retrospectively studied primary lumbar puncture providers in the USA from 1991-2011. In 2011, radiologists performed $46.6 \%$ $(n=45,338)$ of lumbar puncture procedures, a significant increase compared with 1991, where they performed only $11.3 \%(n=10,533)$. This increase was reflected in both therapeutic and diagnostic lumbar puncture. ${ }^{37}$ On the other hand, neurologists and neurosurgeons did $14,453(14.9 \%)$ lumbar puncture procedures in 2011 compared with 46,146 (49.4\%) in 1991, a significant decrease. Trunz et al. found similar results; radiologists held the increasingly largest share of diagnostic and overall lumbar puncture procedures (overall share, $45.7 \%[n=42,296]$ in 2010 versus $52.3 \%[n=48,414]$ in 2018). Emergency medicine physicians and neurologists had a decreasing procedure share between 2010 and 2018 (21.8\% versus $15.3 \%$ and $12.5 \%$ versus $8.8 \%$, respectively). ${ }^{38}$ Hence, radiology has now become the dominant provider of lumbar puncture procedures. ${ }^{37}$

\section{Lumbar puncture on an outpatient basis}

While the number of lumbar puncture procedures being performed remains almost unchanged, there has been an increasing trend towards performing lumbar puncture procedures in the outpatient and emergency settings, with fewer inpatient lumbar puncture procedures $(-37 \%$ in the study by Kroll et al.: 44,817 in 2011 versus 71,385 in 1991). ${ }^{37}$ Outpatient lumbar punctures can reduce the costs and duration of hospitalizations. ${ }^{39}$ However, concern remains regarding the safety and efficacy of outpatient lumbar puncture procedures. Barreras et al. retrospectively evaluated elective lumbar punctures performed at a resident-run university hospital outpatient lumbar puncture clinic between June 2014 and May 2015..$^{0}$ Of the 282 patients who underwent lumbar puncture, CSF was successfully acquired in 267 (94.6\%) patients, and the incidence of post-lumbar puncture headache (PLPH) was $16 \%$, the same as the accepted inpatient complication rate. ${ }^{40}$ However, PLPH incidence was estimated based on patient-reported symptoms, and the majority of those reported were severe. Therefore, the true incidence of PLPH may have been missed. Lambe et al. ${ }^{41}$ and Popp et al..$^{39}$ showed that the complication rate from outpatient lumbar puncture was $4 \%$ and $9 \%$, respectively; complications were mild PLPH and did not require hospitalization. So, a standardized approach via an outpatient lumbar puncture clinic can produce a high rate of success, with low rates of post-procedure complications.

\section{Safety checklist before lumbar puncture}

King's College Hospital, London, UK, provides an outpatient lumbar puncture service for patients throughout south-east England. Previously, many patients attended appointments without a recently documented coagulation profile or full medical history, which made it difficult to assess the risk of cerebral herniation. ${ }^{42}$ As a result, multiple scheduled lumbar punctures were cancelled. To mitigate this, the clinic decided to email the 
Figure 3: Flow chart of contraindications to lumbar puncture

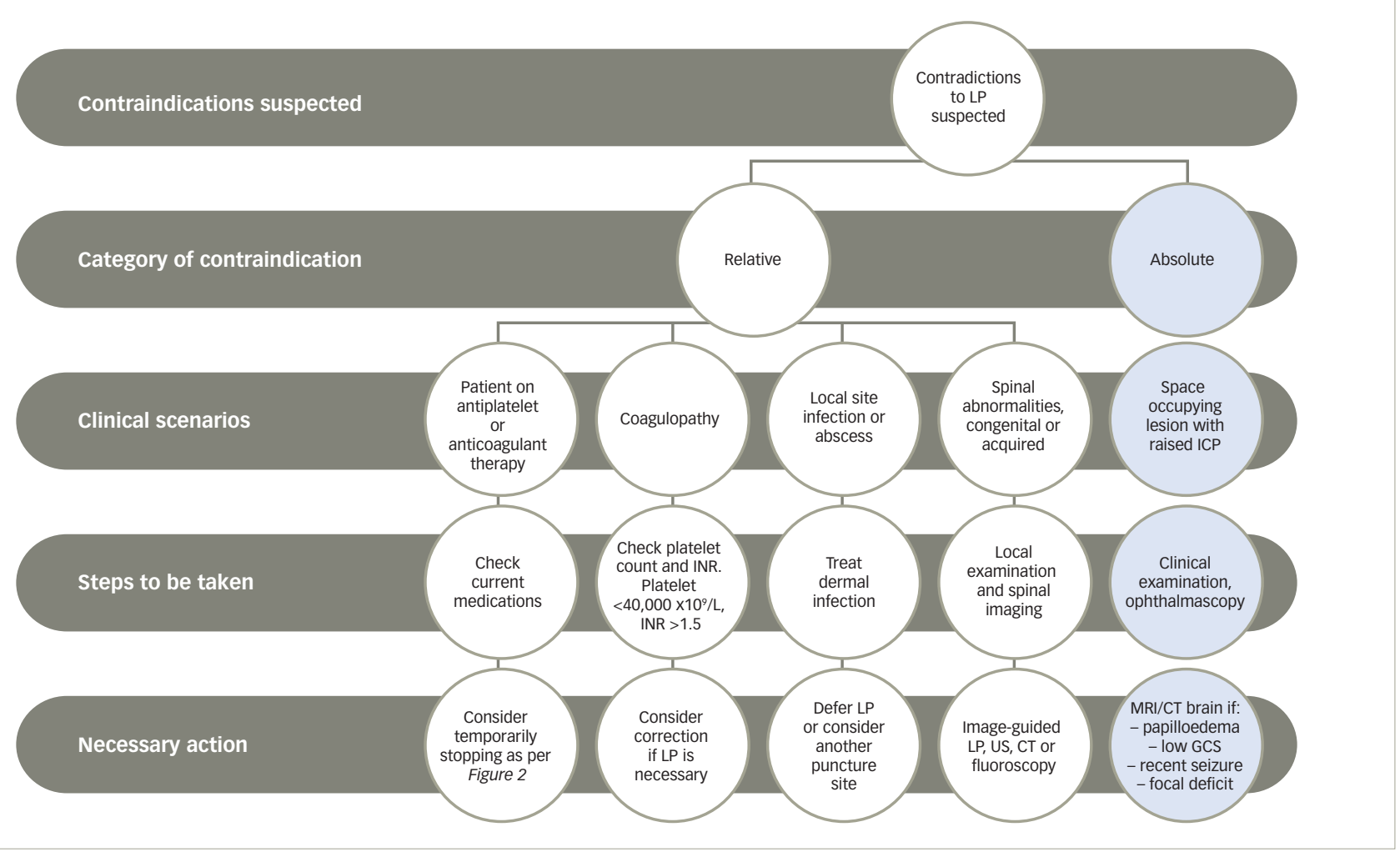

A flow chart showing the contraindications to lumbar puncture and the procedures to follow to rule out these contraindications.

$C T=$ computed tomography; GCS = Glasgow Coma Scale; ICP = intercranial pressure; INR = international normalized ratio; $L P=$ lumbar puncture; $\mathrm{MRI}=$ magnetic resonance imaging; US = ultrasound.

referring neurologist a safety checklist to be completed before referring. The checklist requested documentation of a recent platelet count and coagulation profile, and a statement regarding clinical or radiological assessment of raised CSF pressure. ${ }^{42}$ After implementing this simple checklist, the percentage of patients referred to the clinic with an available platelet count, coagulation profile and intracranial imaging increased to $89 \%, 82 \%$ and $98 \%$, respectively, compared with $25 \%, 18 \%$ and $75 \%$, respectively, before the safety checklist. This increased the efficiency of the clinic, as fewer procedures needed to be cancelled, and indirectly increased the safety of the procedure by increasing the confidence of the junior doctors performing the procedure. ${ }^{42}$ However, lack of a detailed risk assessment prior to lumbar puncture, in a setting of outpatient diagnostic lumbar puncture, was a major limitation of the study.

The 2015 European Society of Clinical Microbiology and Infectious Diseases (ESCMID) guidelines for the diagnosis and treatment of bacterial meningitis strongly recommend imaging the brain before performing lumbar puncture in patients presenting with:43

- focal neurologic deficits (excluding cranial nerve palsies)

- recent seizures

- impaired consciousness (evident as Glasgow Coma Scale of <10)

- known immunocompromised state.

Using inputs from nine experts and applying the modified Delphi technique, Berg et al. suggested a 20-point lumbar puncture checklist, which provides step-by-step guide for before and during the procedure (Figure 4). ${ }^{44}$

\section{Complications of lumbar puncture}

It is widely accepted that lumbar puncture is a safe intervention, yet complications can occur. ${ }^{32}$ The most common complication is PLPH, which is an orthostatic headache caused by CSF leakage, usually starting within 48 hours of the lumbar puncture in $90 \%$ of patients. ${ }^{45}$ In $80 \%$ of patients, PLPH resolves within 7 days, but in a minority, it may persist for weeks or months. ${ }^{46}$ The reported incidence of PLPH varies from $1 \%$ to $50 \% .{ }^{46}$ The patient-related risk factors for PLPH include younger age, past history of headache, female sex and anxiety about post-lumbar puncture complications. ${ }^{32}$ The risk of PLPH can be reduced by using a $25 \mathrm{G}$ atraumatic needle, attempting lumbar puncture fewer than four times, passively withdrawing $\leq 30 \mathrm{~mL}$ CSF and having the patent lie in a lateral recumbent position. ${ }^{32}$ Among these, traumatic (standard) or atraumatic needle tip design constitutes the most important factor. Nath et al. did a meta-analysis of 102 randomized controlled trials, comprising 31,412 subjects, on the occurrence of PLPH. ${ }^{45}$ This study found the incidence of PLPH to be $4.2 \%$ in the atraumatic needle group and $11.0 \%$ in the conventional needle group ( $p<0.0001$, relative risk $0.40,95 \%$ confidence interval [Cl] 0.34-0.47). Compared with the standard needles, there was also decreased need for epidural blood patch, analgesia and post-lumbar puncture hospitalization, with a similar procedural success rate and similar incidence of traumatic lumbar puncture. ${ }^{45}$ Lack of cost-effectiveness analysis between the needle types (which is important for drafting health policy), unequal distribution of patients among the outcome factors analysed and lack of a quantitative assessment of ease of use of atraumatic needles among the clinicians performing lumbar puncture, were major limitations of the study. 


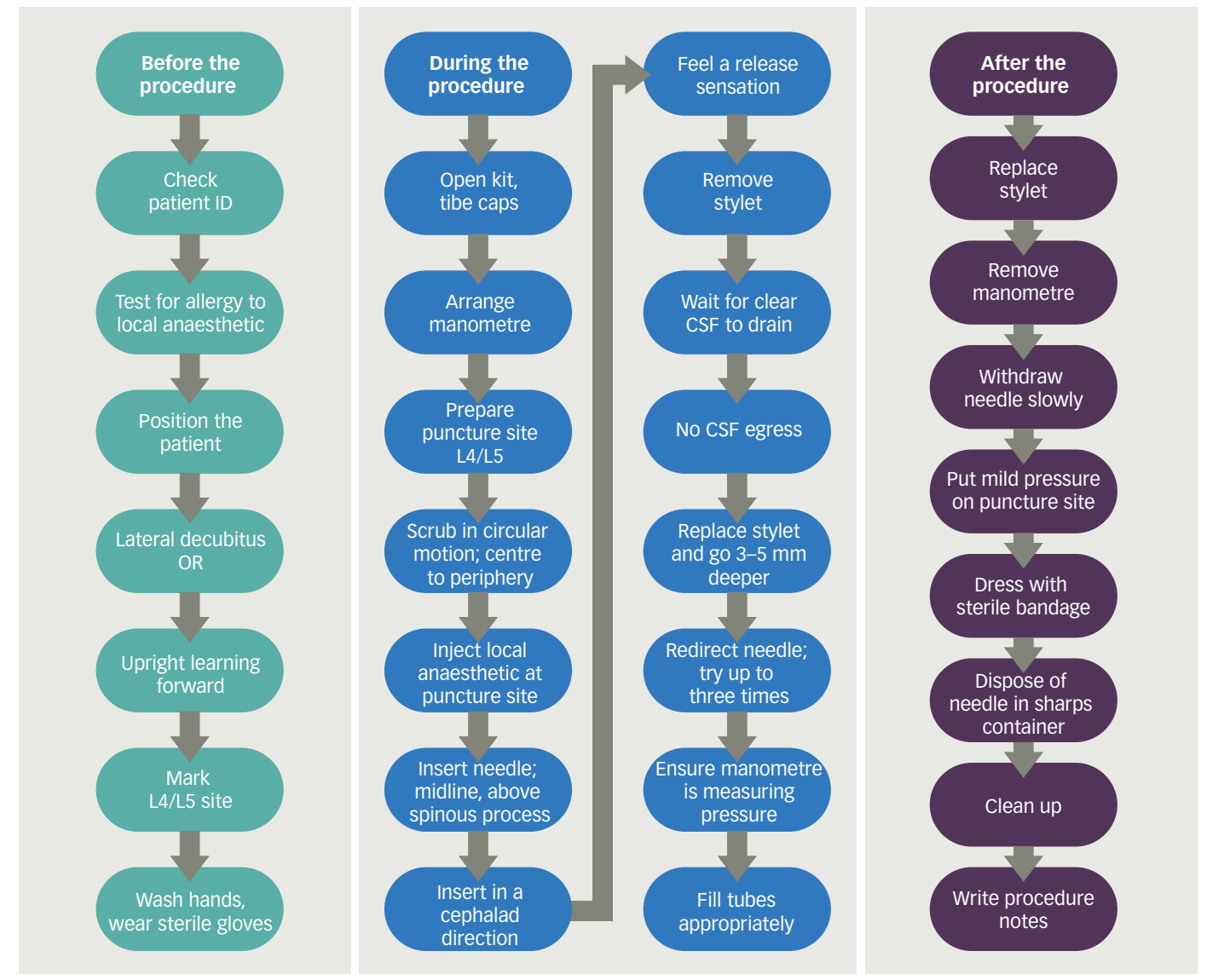

A step-by-step checklist for the physician that can be followed before, during and after the lumbar puncture procedure. CSF = cerebrospinal fluid; ID = identification.

In a retrospective study by Moisset et al., atraumatic needles were used in only $8 \%$ of 6,594 lumbar puncture procedures performed in two French university hospitals in 2014, showing considerable lack of awareness among practitioners. ${ }^{5}$ As PLPH is an orthostatic headache, it is initially treated with mild analgesics and bed rest. ${ }^{45}$ If persistent, caffeine, theophylline and hydrocortisone can be tried, but evidence on their efficacy is lacking. ${ }^{47}$ Evidence does not support bed rest or fluid supplementation for preventing PLPH. ${ }^{48} \mathrm{An}$ epidural blood patch may be considered if PLPH persists for more than 5 days. ${ }^{49}$

Back pain and nerve root irritation occur in $15 \%$ and $11 \%$ of lumbar punctures, respectively; incidence decreases when atraumatic needles are used. ${ }^{45}$ Other rare complications include cerebral herniation (3-7\%), ${ }^{50}$ bacterial meningitis $(<0.1 \%),{ }^{51}$ spinal haematoma (incidence unknown) ${ }^{52}$ and cerebral venous sinus thrombosis (incidence unknown)..$^{53}$

A possible complication, currently being investigated, is accelerated disc degeneration following penetration of the intervertebral disc joint during lumbar puncture. ${ }^{54}$ Ertas et al. evaluated the risk of disc puncture during a standard lumbar puncture procedure in 50 human cadavers. The probability of puncturing the joint was $20 \%$ for $L 3-4,38 \%$ for $L 4-5$ and $16 \%$ for L5-S1. The total probability of disc penetration was $25 \%$. Statistical analysis revealed significantly increased risk of disc penetration when performing lumbar puncture at the $L 4-5$ level compared with $L 5-S 1 \quad(p=0.023)$. This study showed that lumbar puncture carries significant risk of intervertebral disc penetration, which may lead to accelerated disc degeneration; however, further studies are required to confirm these findings. ${ }^{55}$

\section{Intrathecal medications}

Intrathecal nusinersen was approved by the United States Food and Drug Administration for the treatment of spinal muscular atrophy (SMA) in December 2016, ${ }^{56}$ and by the European Medicines Agency in June 2017.57 In 26 patients with SMA, Mousa etal. were able to successfully perform 104 intrathecal nusinersen injections, even in patients with neuromuscular scoliosis or spinal instrumentation (44 of the 104 procedures were performed in 11 such patients). There were no immediate or long-term complications. ${ }^{58}$ Wurster et al. evaluated the practicability and safety of intrathecal nusinersen in adolescent and adult patients with late-onset SMA (SMA type 2 and 3), in whom (roto) scoliosis, previous spine fusion operations, as well as joint contractures and respiratory insufficiency are frequently observed, and make lumbar puncture complicated. They analysed 93 lumbar punctures in 20 such patients and surveyed the duration and site of lumbar puncture, oxygen saturation, number of attempts, need for sedation and analgesia. ${ }^{59}$ Intrathecal nusinersen was found to be safe, feasible and well tolerated (complication rate was $5 \%$ and consisted of mild PLPH), even in patients with adult-onset SMA, in both standard and image-guided lumbar puncture. ${ }^{59}$

Many intracranial infections are smouldering infections, which carry high mortality if not promptly and aggressively treated. ${ }^{60}$ Unfortunately, very few antibiotics cross the blood-brain barrier to reach concentrations high enough to be effective. ${ }^{61,62}$ Intrathecal and intraventricular administration of antibiotics can be lifesaving in such situations. Bargiacchi et al. systematically reviewed 51 case studies of adults with gram-negative CNS infections treated with intrathecal or intraventricular 
Table 1: Reported case series on intrathecal interferon in patients with subacute sclerosing panencephalitis and their dosing and outcome

\begin{tabular}{|c|c|c|c|c|}
\hline Study & Patients & Dosage & Follow-up & Result \\
\hline Anlar, et al. 66 & 22 & $1 \mathrm{MU} /$ week & $4-9$ years & $\begin{array}{l}50 \% \text { remission, } \\
22 \% \text { stabilized, } \\
28 \% \text { progressed }\end{array}$ \\
\hline Miyazaki, et al. ${ }^{67}$ & 1 & $3 \mathrm{MU} /$ week & 18 years & Remission for 8 years \\
\hline Panitch, et al. ${ }^{65}$ & $\begin{array}{l}3 \\
(\text { stage } 2 / 3)^{*}\end{array}$ & Not reported & 2 years & $100 \%$ remission \\
\hline Steiner, et al. ${ }^{68}$ & 3 & $1 \mathrm{MU}$ twice weekly & 2 years & $100 \%$ remission \\
\hline Kurata, et al. ${ }^{69}$ & 1 (stage 2) & $3 \mathrm{MU} /$ week & 6 months & Improved \\
\hline Horiguchi, et al. ${ }^{70}$ & 1 & $6 \mathrm{MU} /$ week & 4 years & improved \\
\hline Moodley, et al. ${ }^{20}$ & 1 & $\begin{array}{l}1.5 \mathrm{MU} \text { on alternate days for } \\
6 \text { weeks }\end{array}$ & 30 months & $\begin{array}{l}\text { Initial improvement then died at } 30 \\
\text { months }\end{array}$ \\
\hline
\end{tabular}

*Stages of subacute sclerosing panencephalitis:50

- Stage 1 - neurological symptoms, such as personality changes, lethargy, difficulty in school and strange behaviour

- Stage 2 - myoclonus, worsening dementia and long-tract motor or sensory disease

- Stages 3 - neurologic deterioration, with eventual flaccidity or decorticate rigidity, and symptoms and signs of autonomic dysfunction; myoclonus is absent

- Stage 4 - vegetative state.

$\mathrm{MU}=$ million units.

Table 2: Proven and exploratory cerebrospinal fluid biomarkers for neurodegenerative diseases ${ }^{70}$

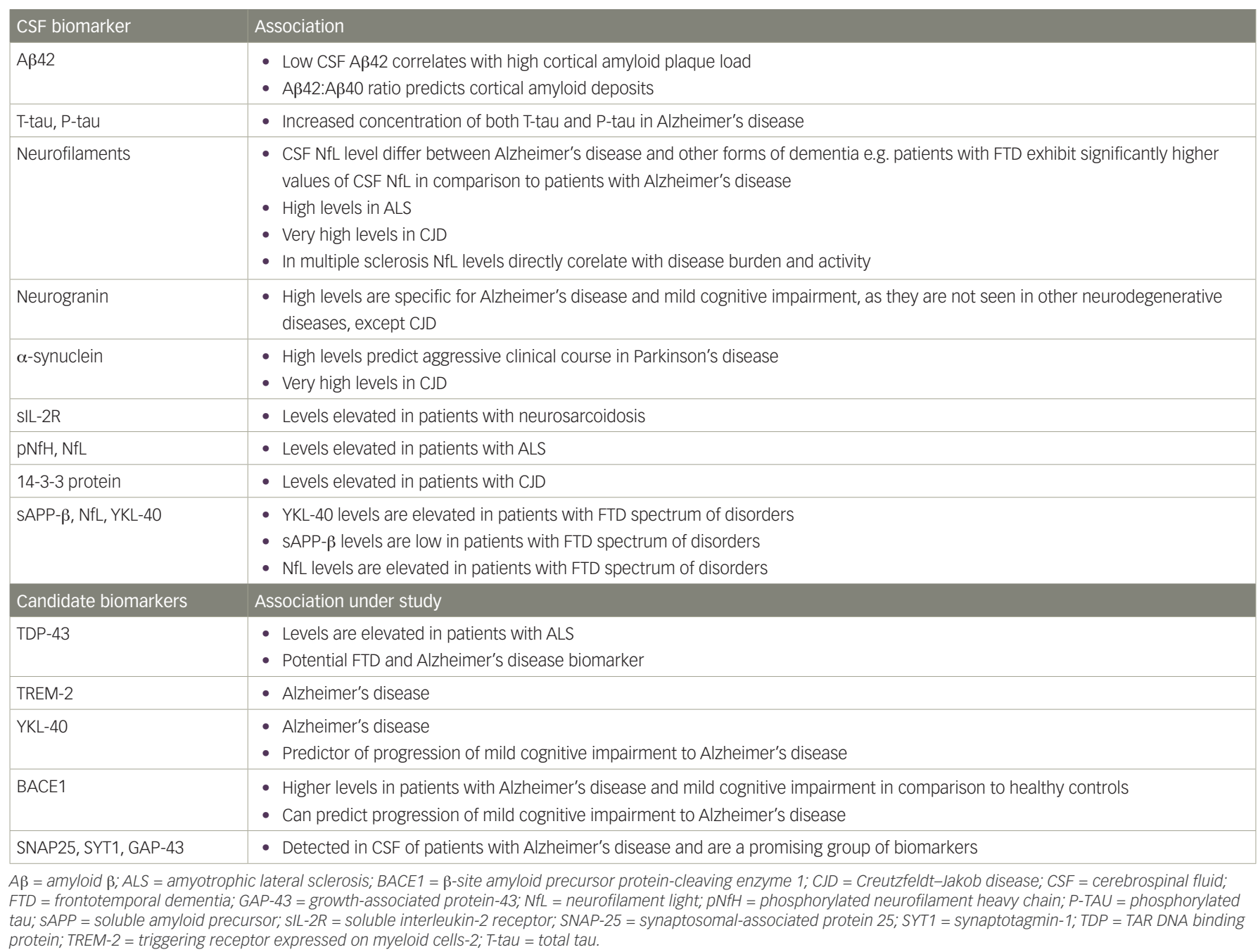




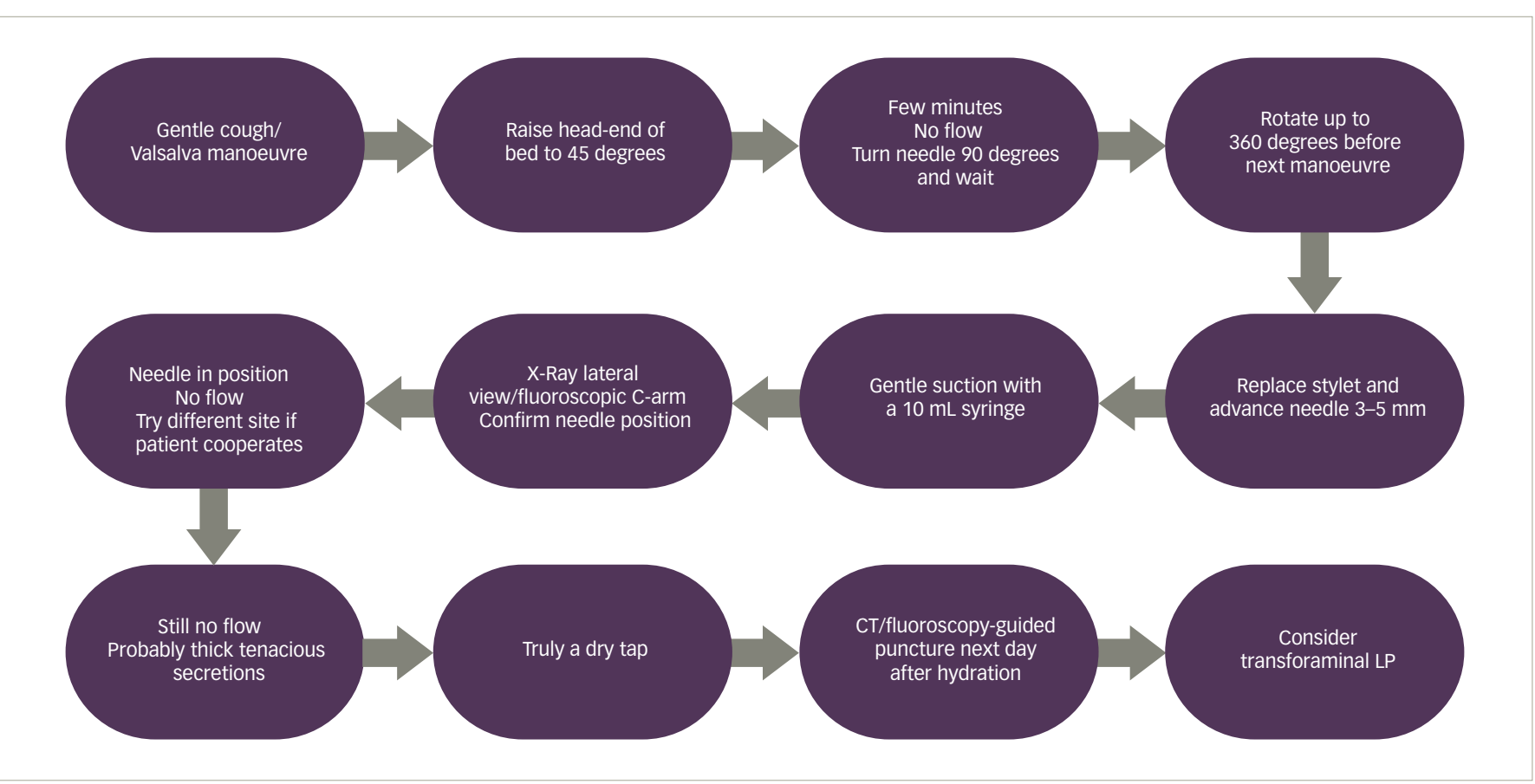

A flowchart showing the manoeuvres that can be tried in the case of failed lumbar puncture - dry tap. The sequence shows the next step to take when the previous one fails to elicit CSF.

CSF = cerebrospinal fluid; $C T=$ computed tomography; $L P=$ lumbar puncture.

colistin. ${ }^{19}$ They found that intrathecal or intraventricular colistin at a dose of 125,000 IU, as suggested by the Infectious Diseases Society of America (IDSA) guidelines, administered once daily for at least 14 days is safe and effective. No nephrotoxicity was reported with intrathecal or intraventricular colistin. ${ }^{19}$ There is no standard criterion for intrathecal or intraventricular vancomycin dosing. Wombwell et al. systematically reviewed cases that reported on intrathecal/intraventricular vancomycin, and recommended that a daily dose of $10 \mathrm{mg}$, aiming for a trough level of $15-20 \mathrm{mg} / \mathrm{L}$, is safe and effective. ${ }^{63}$

Various other combinations of antibiotics have been administered intrathecally. Zhang et al. reported 86 patients with intracranial infections with severe traumatic brain injury. ${ }^{64}$ The group treated with intrathecal meropenem and vancomycin had better cure time than patients treated with intravenous meropenem and vancomycin $(p=0.004)$, and experienced fewer adverse reactions $(p=0.035)$ and fewer severe sequelae $(p=0.007) .{ }^{64}$

Though it has been more than three decades since Panitch et al. injected interferon into CSF to treat subacute sclerosing panencephalitis for the first time in 1986, there are still no concrete guidelines as to the dose and dosing schedule of intrathecal interferon. ${ }^{65}$ Studies detailing their dosing schedule are shown in Table 1.20,66-70 Based on studies by Steiner et al., Kurata et al. and Thurner et al., we recommend continuous intrathecal infusion of interferon at a rate of 3 million U/week as more effective than intermittent bolus infusion. ${ }^{68,69,71}$

\section{Newer advances in cerebrospinal fluid diagnostics}

Alongside the common diagnostic uses of CSF (detailed above in Common indications for lumbar puncture), rapid advances in diagnostic techniques have allowed CSF to be used in diagnosing various other neurodegenerative and neuro-immunomodulatory disorders. The CSF diagnostic or prognostic biomarkers in development for various neurodegenerative and neuro-immunomodulatory diseases are shown in Table $2 .^{72}$

\section{Difficult lumbar puncture}

Standard lumbar puncture without image guidance is routinely performed by neurologists or an internal medicine physician. However, for trainees early in their career the successful performance of standard lumbar puncture procedure can be difficult, especially in uncooperative, immobilized or obese patients, or patients undergoing spine surgery. ${ }^{73,74}$ When neurologists or internal medicine physicians performing a bedside standard lumbar puncture procedure encounter a difficult-to-access CSF space and a dry tap, they can follow the step-by-step manoeuvres outlined in Figure $5 .^{74}$

\section{Fluoroscopy-guided lumbar puncture}

Non-image-guided standard lumbar puncture procedures are more likely to result in traumatic tap, which may affect laboratory results, potentially leading to elevated cell counts and CSF protein levels. ${ }^{36}$ Obesity (causing inability to identify or palpate osseous landmarks routinely used to plan standard lumbar puncture, i.e. spinous processes or the iliac crest) and/or degenerative disc disease are being seen in an increasing number of patients, potentially leading to an increase in failed bedside non-image-guided standard lumbar puncture procedures. ${ }^{75}$ Also, as lumbar puncture is highly operator dependent, the fear of a potential negligence suit has persuaded physicians to refer such procedures to radiologists. ${ }^{37}$ Lumbar puncture under imaging guidance is usually preferred when there is postoperative hardware and/or osseous fusion, extensive degenerative change or scoliosis. ${ }^{36}$ 
As mentioned above (see common indications of lumbar puncture), there are more intrathecal medications, many of which are expensive (e.g. intrathecal nusinersen for SMA costs around US\$708,000 / year): ${ }_{i}^{76}$ therefore, confirmatory imaging with intrathecal administration is preferred, promoting use of image-guided lumbar puncture. ${ }^{36}$ All these factors combined have led to increasing requests for image-guided lumbar punctures for both diagnostic and therapeutic procedures, especially fluoroscopy-guided lumbar puncture. Fluoroscopy-guided lumbar puncture is considered safe and efficacious, ${ }^{75}$ with a mean effective radiation dose of $(n=2.9 \mathrm{mSv})^{77}$ and an acceptable rate of complications: $2.2 \%$ of patients develop PLPH, and only $0.8 \%$ require an epidural blood patch. ${ }^{78}$ Nerve root irritation and infectious complications have not been reported, even from centres that perform 1,000 fluoroscopy-guided lumbar punctures per year.77,78

\section{Meningeal enhancement post-lumbar puncture}

In clinical practice, the concern for iatrogenic meningeal enhancement following lumbar puncture is a common reason for performing contrast enhanced brain MRI prior to lumbar puncture. This practice, however, may lead to delayed diagnosis. ${ }^{79}$ Review of the literature reveals that there are only two studies, to date, investigating whether uncomplicated lumbar puncture procedure can cause meningeal enhancement. ${ }^{79,80}$ In 1994, Mittl et al. performed an ambispective study of patients who had a lumbar puncture within the 30 days prior to a contrasted brain MRI However, they did not compare their cases with a control group. Only two cases had meningeal enhancement on MRI (1/11 in the retrospective group, 1/97 in the prospective group) where lumbar puncture was performed 30 days prior to contrasted MRI. However, traumatic lumbar puncture and intracranial hypotension arguably explained these two cases of pachymeningeal enhancement..$^{80}$

In a retrospective study, Wesley et al. analysed contrast enhancement on brain MRI in patients who had lumbar puncture before imaging $(n=77)$ compared with controls, in whom lumbar puncture was not done before MRI $(n=707) .{ }^{79}$ Of the 77 patients who received lumbar puncture, only one had unexplained meningeal enhancement (1.2\%). Of the 36 patients in the control group who had enhancement, none were unexplained $(p=0.098) .^{79}$ However, the authors did not match cases and controls one-to-one with similar patient characteristics, such as age and sex. Also, given the low rate of event occurrence (meningeal enhancement), a larger sample size in the case group would have been appropriate to avoid type II error in this study. Deferring lumbar puncture until after brain MRI may not be helpful in situations where performing lumbar puncture is vital.

\section{Computed tomography of the head before lumbar puncture in meningitis: Excessive or due diligence?}

It has become the norm to routinely perform brain imaging in patients with suspected meningitis. Gopal et al. and Hasbun et al. studied 111 and 301 patients, respectively, and found that performing computed tomography (CT) before lumbar puncture in patients with suspected meningitis with the following clinical features resulted in a 31.5\% and $41 \%$ reduction in the number of CTs: age $>60$ years, history of CNS disease, severe immunocompromise, impaired consciousness, new onset seizures or focal neurological deficits. ${ }^{81,82}$ This also translated into a shorter time between the patient being admitted and the first dose of antibiotic, which can have a significant impact on mortality. ${ }^{83}$ Furthermore, the diagnostic-treatment sequence: head CT followed by lumbar puncture, then antibiotics increases the mortality rate (odds ratio 5.6). ${ }^{83}$ An IDSA survey in 2016 also noted that, instead of adhering to the guidelines, most physicians order imaging before performing lumbar puncture in patients with suspected meningitis, thereby delaying diagnosis and administration of antibiotics, and increasing in-hospital expenditure. ${ }^{84}$ Only patients with suspected meningitis who present with the clinical features discussed above should undergo advanced imaging before lumbar puncture, instead of routinely imaging every patient before lumbar puncture.

\section{Conclusions}

Lumbar puncture is a widely performed procedure that is done across all levels of care, without frequent complications. However, caution is advised when performing lumbar puncture in patients at high risk of cerebral herniation or with severe coagulopathy. ${ }^{32}$ A checklist before lumbar puncture helps to improve physician's confidence and avoid complications. ${ }^{42}$ With the advent of intrathecal medications and sophisticated spinal protheses, and the increase in the number of difficult lumbar punctures encountered by physicians, demand for image-guided lumbar punctures has risen. ${ }^{75}$ There is convincing evidence that an atraumatic needle tip results in fewer complications; therefore, emphasizing the need for more awareness of this technique among physicians. ${ }^{45}$ Outpatient lumbar puncture is as effective and safe as inpatient lumbar puncture, and can help reduce the burden of admission and cost. ${ }^{40}$ Newer CSF biomarkers may pave the way for earlier diagnosis of neurodegenerative diseases. ${ }^{85}$ The practice of deferring lumbar puncture until after brain MRI may not be helpful in situations where performing lumbar puncture is vital. ${ }^{79}$ Certain clinical features should warrant performing imaging before lumbar puncture in suspected meningitis, rather than routinely performing imaging in every patient before lumbar puncture. •
McLane HC, Berkowitz AL, Patenaude BN, et al. Availability, accessibility and affordability of neurodiagnostic tests in 37 countries. Neurology. 2015;85:1614-22.

Pople IK. Hydrocephalus and shunts: what the neurologist should know. J Neurol Neurosurg Psychiatry. 2002:73(Suppl. 1):i17.

3. Khasawneh AH, Garling RJ, Harris CA. Cerebrospinal fluid circulation: What do we know and how do we know it? Brain Circ. 2018:4:14-18.

4. Sakka L, Coll G, Chazal J. Anatomy and physiology of cerebrospinal fluid. Eur Ann Otorhinolaryngol Head Neck Dis. 2011;128:309-16.

5. Moisset X, Ruet A, Brochet B, et al. who performs lumbar puncture, how many do they perform, how and why? A retrospective study of 6,594 cases. Eur Neurol. 2016;76:8-11. MCGill F, Heyderman RS, Panagiotou S, et al. Acute bacterial meningitis in adults. Lancet. 2016;388:3036-47.

Su G, Fu Z, Hu L, et al. $16 \mathrm{~S}$ ribosomal ribonucleic acid gene polymerase chain reaction in the diagnosis of bloodstream infections: A systematic reviow and meta analysis. PLOS One. 2015:10:e0127195

8. Oya AL Chapter Eleven - Direct Application of MALDI-TOF Mass Spectrometry to Cerebrospinal Fluid for Pathogen Identification. In: Cobo F (eds). The Use of Mass Spectrometry Technology (MALDI-TOF) in Clinical Microbiology. Cambridge Technology (MALDI-TOF) in Clinical Microbio

MA, USA: Academic Press, 2018;159-65.
Salvarani C, Brown Jr RD, Hunder GG. Adult primary centra nervous system vasculitis. Lancet. 2012;380:767-77.

10. Graus F, Titulaer MJ, Balu R, et al. A clinical approach to diagnosis of autoimmune encephalitis Lancet Neurol. 2016;15:391-404.

11. Kupila L, Jaakonmäki $\mathrm{N}$, Huhtala $\mathrm{H}$, Airas L. Acute transverse myelitis: CSF determinants in the differential diagnostics. Neurology. 2014;82(10 Suppl.):P5.180

12. Illes Z, Blaabjerg M. Cerebrospinal fluid findings in Guillain-Barré syndrome and chronic inflammatory demyelinating polyneuropathies. Handb Clin Neurol. 2017;146:125-138

3. Beh SC, Greenberg BM, Frohman T, Frohman EM. Transvers myelitis. Neurol Clin. 2013;31:79-138.

14. Deisenhammer F, Zetterberg H, Fitzner B, Zettl UK. The cerebrospinal fluid in multiple sclerosis. Front Immunol. 2019;10:726

5. Macdonald RL, Schweizer TA. Spontaneous subarachnoid haemorrhage. Lancet. 2017;389:655-66.

16. Subirá D, Serrano C, Castañón S, et al. Role of flow cytometry immunophenotyping in the diagnosis of leptomeningeal carcinomatosis. Neuro Oncol. 2012;14:43-52.

7. Jensen RH, Radojicic A, Yri H. The diagnosis and management of idiopathic intracranial hypertension and the associated of idiopathic intracranial hypertension and the associa

18. Ishikawa M, Hashimoto M, Mori E, et al, The value of the cerebrospinal fluid tap test for predicting shunt effectiveness in idiopathic normal pressure hydrocephalus. Fluids Barriers CNS. 2012;9:1.
19. Bargiacchi O, Rossati A, Car P, et al. Intrathecal/intraventricular colistin in external ventricular device-related infections by multi-drug resistant Gram negative bacteria: case reports and review. Infection. 2014:42:801-9.

20. Moodley K, Bill P, Patel VB. Interferon alpha in subacute sclerosing panencephalitis: Case report and review of the sclerosing pant, 2015;31:130-4.

21. Veerapandiyan $A$, Eichinger $K$, Guntrum $D$, et al. Intrathecal nusinersen in older children and adults with spinal muscular atrophy. Neurology. 2019;92(15 Suppl.):S5.001.

22. Clement JM, Holle LM. Safe Administration of intracerebral spinal fluid chemotherapy: time for guidelines. J Oncol Pract. 2017;13:713-18

23. Hasnat MJ, Rice JE. Intrathecal baclofen for treating spasticity in children with cerebral palsy. Cochrane Database Syst Rev. 2015;CD004552

24. Raza SM, Banu MA, Donaldson A, et al. Sensitivity and specificity of intrathecal fluorescein and white light excitation for detecting intraoperative cerebrospinal fluid leak in endoscopic skull base surgery: a prospective study. endoscopic skul base surgery: a

25. Abassi M, Boulware DR, Rhein J. cryptococcal meningitis: diagnosis and management update. Curr Trop Med Rep. 2015;2:90-9.

26. Rolfes MA, Hullsiek KH, Rhein J, et al. The effect of therapeutic lumbar punctures on acute mortality from cryptococcal 
meningitis. Clin Infect Dis. 2014;59:1607-14.

27. Mollan SP, Davies B, Silver NC, et al. Idiopathic intracranial hypertension: consensus guidelines on management. J Neurol Neurosurg Psychiatry. 2018;89:1088-100.

28. Figueiredo AHA, Brouwer MC, van de Beek D. Acute community-acquired bacterial meningitis. Neurol Clin. 2018;36:809-20

29. Joffe AR. Lumbar puncture and brain herniation in acute bacterial meningitis: a review. I Intensive Care Med. 2007;22:194-207.

30. Krishnan P, Roychowdhury S. Spinal coning after lumbar puncture in a patient with undiagnosed giant cervica neurofibroma. Ann Indian Acad Neurol. 2013;16:440-2.

31. van de Beek D, de Gans J, Tunkel AR, Wijdicks EFM. Community-acquired bacterial meningitis in adults. N Engl J Med. 2006;354:44-53

32. Engelborghs $\mathrm{S}$, Niemantsverdriet $\mathrm{E}$, Struyfs $\mathrm{H}$, et al. Consensus guidelines for lumbar puncture in patients with neurological diseases. Alzheimers Dement (Amst). 2017:8:111-26.

33. Gogarten $W$, Vandermeulen $E$, Van Aken $\mathrm{H}$, et al. Regional anaesthesia and antithrombotic agents: recommendations of the European Society of Anaesthesiology. Eur J Anaesthesiol. 2010;27:999-1015

34. Lee PW, Levy M. Risk of hematoma from aspirin or clopidogrel owing to lumbar puncture. Mayo Clin Proc. 2019;94:1552-5.

35. Bodilsen J, Mariager T, Vestergaard HH, et al. Association of lumbar puncture with spinal hematoma in patients with and without coagulopathy. JAMA. 2020;324:1419-28.

36. Eskey CJ, Ogilvy CS. Fluoroscopy-guided lumbar puncture: decreased frequency of traumatic tap and implications for the assessment of CT-negative acute subarachnoid hemorrhage. AJNR Am J Neuroradiol. 2001;22:571-6.

37. Kroll H, Duszak R, Nsiah E, et al. Trends in lumbar puncture over 2 decades: A dramatic shift to radiology. AJR Am I Roentgenol. 2015;204:15-9.

38. Trunz $L M$, Gandhi AV, Karambelkar AD, et al. National trends in lumbar puncture from 2010 to 2018: A shift reversal from the emergency department to the hospital setting for radiologists and advanced practice providers. AJNR Am J Neuroradiol. 2021;42:206-10.

39. Popp J, Riad M, Freymann K, Jessen F. [Diagnostic lumbar puncture performed in the outpatient setting of a memory clinic. Frequency and risk factors of post-lumbar puncture headache] [Article in German]. Nervenarzt. 2007;78:547-51.

40. Barreras P, Benavides DR, Barreras JF, et al. A dedicated lumbar puncture clinic: performance and short-term patient outcomes. J Neurol. 2017;264:2075-80.

41. Lambe J, Redmond J. Analysis of the outpatient lumbar puncture service in a tertiary hospital Ir I Med Sci. 2018;187:521-4.

42. Sweeney M, Al-Diwani A, Hadden R. Improving the safety and efficiency of outpatient lumbar puncture service. BMJ Qual Improv Report. 2016:5.4629.w4412.

43. van de Beek D, Cabellos C, Dzupova O, et al. ESCMID guideline: diagnosis and treatment of acute bacterial meningitis. Clin Microbiol Infect. 2016;22(Suppl. 22):S37-62.

44. Berg K, Riesenberg LA, Berg D, et al. The development of validated checklist for adult lumbar puncture: preliminary results. Am J Med Qual. 2013;28:330-4

45. Nath S, Koziarz A, Badhiwala JH, et al. Atraumatic versus conventional lumbar puncture needles: a systematic review and meta-analysis. Lancet. 2018;391:1197-204.

46. Amorim JA, Gomes de Barros MV, Valença MM. Post-dura (post-lumbar) puncture headache: Risk factors and clinical features. Cephalalgia. 2012:32:916-23.

47. Basurto Ona X, Martínez García L, Solà I, Bonfill Cosp X
Drug therapy for treating post-dural puncture headache Cochrane Database Syst Rev 2011:CD007887.

48. Arevalo-Rodriguezl, Ciapponi A, Roqué i Figuls M, et Posture and fluids for preventing post-dural puncture headache Cochrane Database Syst Rev. 2016:3:CD00919.

49. van Kooten F Oedit R, Bakker SIM, Dippel DWJ. Epidural blood patch in post dural puncture headache: a randomised, observer-blind, controlled clinical trial. J Neurol Neurosurg Psychiatry. 2008;79:553-8.

50. Brouwer MC, Coutinho JM, van de Beek D. Clinical characteristics and outcome of brain abscess: Systematic review and meta-analysis. Neurology. 2014;82:806-13.

51. Durand ML, Calderwood SB, Weber DJ, et al. Acute bacterial meningitis in adults - a review of 493 episodes. N Eng/ J Med. 1993;328:21-8

52. Van Veen JJ, Nokes TJ, Makris M. The risk of spinal haematoma following neuraxial anaesthesia or lumbar puncture in thrombocytopenic individuals: review Br 1 Haematol. 2010;148:15-25

53. Kate MP, Thomas B, Sylaja PN. Cerebral venous thrombosis in post-lumbar puncture intracranial hypotension: case report and review of literature. F1000Res. 2014:3:41

54. Moinuddin FM, Wahood W, Yolcu Y, et al. Lumbar puncture ncreases risk of lumbar degenerative disc disease: analysis from the rochester epidemiology project. Spine. 2020;45:E1326.

55. Ertas A, Gediz T, Ozdol C, et al. Risk of intervertebral disc join puncture during lumbar puncture. Clin Neurol Neurosurg. 2021;200:106107

56. US Food and Drug Administration. Prescribing Information. SPINRAZA (nusinersen) injection, for intrathecal use. 2016. Available at: www.accessdata.fda.gov/drugsatfda_docs/ label/2016/209531 lbl.pdf (accessed 19 July 2021).

57. European Medicines Agency. Spinraza. Summary of Product Characteristics. 2017. Available at: wumwema europa ew/ en/documents/product-information/spinraza-epar-productinformation en pdf (accessed 19 July 2021).

58. Mousa MA, Aria DJ, Schaefer CM, et al. A comprehensive institutional overview of intrathecal nusinersen injections for spinal muscular atrophy. Pediatr Radiol. 2018;48:1797-805.

59. Wurster $C D$, Winter B, Wollinsky K, et al. Intrathecal administration of nusinersen in adolescent and adult SMA typ 2 and 3 patients. J Neurol. 2019;266:183-94.

60. Robertson FC, Lepard JR, Mekary RA, et al. Epidemiology of central nervous system infectious diseases: a meta-analysis and systematic review with implications for neurosurgeons worldwide. J Neurosurg. 2018;1-20. doi: 10.3171/2017.10. JNS17359. Online ahead of print

61. Nau R, Sörgel F, Eiffert H. Penetration of drugs through the blood-cerebrospinal fluid/blood-brain barrier for treatment of central nervous system infections. Clin Microbiol Rev. 2010;23:858-83.

62. Dadparvar M, Wagner S, Wien S, et al. HI 6 human serum albumin nanoparticles--development and transport over an in vitro blood-brain barrier model. Toxicol Lett. 2011:206:60-6.

63. Wombwell E, Young N. Intrathecal and intraventricular vancomycin: a literature review. Infectious Diseases in Clinica Practice. 2014;22:141-4.

64. Zhang Q, Chen H, Zhu C, et al. Efficacy and safety of intratheca meropenem and vancomycin in the treatment of postoperative intracranial infection in patients with severe traumatic brain injury. Exp Ther Med. 2019;17:4605-9.

65. Panitch H, Gomez-Plascencia J, Norris F, et al. Subacute sclerosing panencephalitis: remission after treatment with intraventricular interferon. Neurology 1986:36:562-6.

66. Anlar B, Yalaz K, Oktem F, Kose G. Long-term follow-up of patients with subacute sclerosing panencephalitis treated with intraventricular alpha-interferon. Neurology. 1997;48:526-8.

67. Miyazaki M, Nishimura $M$, Toda $Y$, et al. Long-term follow-up of a patient with subacute sclerosing panencephalitis successfully treated with intrathecal interferon alpha. Brain Dev 2005:27:301-3.

68. Steiner I, Wirguin I, Morag A, Abramsky O. Intraventricular interferon treatment for subacute sclerosing panencephalitis. I Child Neurol. 1989;4:20-4.

69. Kurata T, Matsubara E, Yokoyama M, et al. Improvement of SSPE by intrathecal infusion of $\alpha$-IFN. Neurology. 2004;63:398-9.

70. Horiguchi Y, Ohya T. Successful treatment of subacute sclerosing panencephalitis with long-term intrathecal large dose of alpha-interferon--a case report. [Article in Japanese]. No To Hattatsu. 1995;27:231-7.

71. Thurner B, Spangenberg P, Kleines M, et al. Continuous intrathecal interferon alpha application in subacute sclerosing panencephalitis. Pediatr Infect Dis I. 2007:26:863.

72. Obrocki P, Khatun A, Ness D, et al. Perspectives in fluid biomarkers in neurodegeneration from the 2019 biomarkers in neurodegenerative diseases course-a joint PhD student course at University College London and University of Gothenburg. Alz Res Therapy. 2020:12:20.

73. Sahebkar-Moghaddam F, Adornato BT. The failed lumbar puncture. Neurology. 2005;64:E24.

74. Hudgins PA, Fountain AJ, Chapman PR, Shah LM. Difficult lumbar puncture: pitfalls and tips from the trenches. AJNR Am I Neuroradiol. 2017;38:1276-83.

75. Özütemiz C, Rykken JB. Lumbar puncture under fluoroscopy guidance: a technical review for radiologists. Diagn Interv Radiol. 2019;25:144-56.

76. Pharmacoeconomic Review Report: Nusinersen (Spinraza): (Biogen Canada Inc.): Indication: Treatment of patients with 50 SMA [Internet]. Ottawa (ON): Canadian Agency for Drugs and Technologies in Health; 2018 Jan. Appendix 1, Cost Comparison. Available at: www.ncbi.nlm.nih.gov/books/NBK534119/ (accessed 8 June 2021).

77. Brook AD, Burns J, Dauer E, et al. Comparison of CT and fluoroscopic guidance for lumbar puncture in an obese population with prior failed unguided attempt. NeuroIntervent Surg. 2014;6:323-7.

78. Rodriguez D, Branstetter BF, Agarwal V, et al. JOURNAL CLUB: Incidence of complications following fluoroscopically guided lumbar punctures and myelograms. AJR Am J Roentgenol. 2016;206:20-5

79. Wesley SF, Garcia-Santibanez R, Liang J, Pyburn D. Incidence of meningeal enhancement on brain MRI secondary to lumbar puncture. Neurol Clin Pract. 2016;6:315-20.

80. Mittl RL, Yousem DM. Frequency of unexplained meningea enhancement in the brain after lumbar puncture. AJNR AM I Neuroradiol. 1994:15:633-8.

81. Gopal AK, Whitehouse JD, Simel DL, Corey GR. Crania computed tomography before lumbar puncture: a prospective clinical evaluation. Arch Intern Med. 1999:159:2681-5.

82. Hasbun R, Abrahams J, Jekel J, Quagliarello VJ. Computed tomography of the head before lumbar puncture in adults with suspected meningitis. N Eng/ J Med. 2001;345:1727-33.

83. Proulx N, Fréchette D, Toye B, et al. Delays in the administration of antibiotics are associated with mortality from adult acute bacterial meningitis. QJM. 2005;98:291-8.

84. Salazar L, Hasbun R. Cranial imaging before lumbar puncture in adults with community-acquired meningitis: clinical utility and adherence to the Infectious Diseases Society of America Guidelines. Clin Infect Dis. 2017;64:1657-62.

85. Costerus JM, Brouwer MC, van de Beek D. Technological advances and changing indications for lumbar puncture in neurological disorders. Lancet Neurol. 2018:17:268-78. 